\title{
Joint Design of Twin-Atennna Assisted Space-Time Multilevel Sphere Packing Aided Coded Modulation
}

\author{
R. Y. S. Tee, O. Alamri and ${ }^{1}$ L. Hanzo \\ School of ECS, University of Southampton, SO17 1BJ, UK. \\ Tel: +44-23-8059 3125, Fax: +44-23-8059 4508 \\ Email: ${ }^{1}$ lh@ecs.soton.ac.uk, http://www-mobile.ecs.soton.ac.uk
}

\begin{abstract}
A new multilevel coding (MLC) scheme invoking sphere packing $(\mathrm{SP})$ modulation is proposed, where the SP arrangement is jointly designed with a space time block code (STBC) for the sake of attaining an additional space diversity gain. An iterative multistage decoding (MSD) aided multilevel sphere packing demodulator is proposed for attaining a high decoding performance at a low decoding complexity, when communicating over a correlated Rayleigh channel. The appropriate design of a 4-dimensional (4D) sphere packing bit-to-SP-symbol mapping scheme and the beneficial choice of the individual coding rates of the MLC scheme allows us to provide an unequal error protection capability, which is often required for efficient audio or video transmissions.
\end{abstract}

\section{INTRODUCTION}

The philosophy of Multilevel Coding (MLC) was first proposed by Imai and Hirawaki [1] for protecting each bit of nonbinary modulated signals with the aid of potentially differentrate binary codes. However, their decoding using a full maximum likelihood detector has an excessive complexity in practical systems [2]. Therefore, Multistage Decoding (MSD) invoking the so-called capacity rules of [2] was introduced as an alternative design option. The highest protection bit $i$ of code $C^{i}$ is decoded first by simultaneously exploiting the $a$ priori information obtained from demodulator, before activating the decoder of the code $C^{i+1}$ etc, where each constituent code of the MLC scheme are decoded individually. Hence, MLC constitutes an efficient coded modulation scheme, having flexible component code rates and low complexity. Furthermore, MLCs are also capable of providing unequal error protection for speech, audio or video systems.

A witty and appealingly low-complexity transmit diversity scheme employing two transmit antennas was proposed by Alamouti [3]. The benefits of Alamouti's transmit diversity scheme inspired Tarokh et al. [4] to generalize this transmission scheme to an arbitrary number of transmit antennas, leading to the general concept of Space-Time Block Codes

The financial support of the EPSRC, UK, Ministry of Higher Education of Saudi Arabia and that of the European Union under the auspices of the Phoenix and Newcom projects is gratefully acknowledged.
(STBC). The beneficial concatenation of MLC and STBC arrangement promises a significant performance improvement for transmissions over Rayleigh fading channels.

Various 2-dimensional (2D) bit-to-symbol mapping schemes have been developed for the sake of improving the achievable MLC performance in terms of its overall bit error ratio (BER) performance [5] [6] and [7]. In this paper, we invoke a sphere packing (SP) modulator [8] for mapping the binary bits of a MLC encoder to multi-demensional (M-D) symbols, which are fed into the STBC encoder for transmission over Rayleigh fading channels, with the advantage of providing spatial diversity. The idea of combining the sphere packing concept with orthogonal transmit diversity design was introduced by Su et al. [8]. The benefits of orthogonal transmit diversity design depend on the minimum Euclidean distance of the transmitted complex symbols, which were further investigated by Alamri et al.[9]. The basic concept of sphere packing may also be employed for designing different constellations in the multi-dimensional Euclidean space, while maintaining a certain minimum Euclidean distance. The SP concept may also be readily combined with the MLC structure for mapping its individual bits to an $M$ dimensional SP space, before feeding the SP symbols to the STBC encoder. We refer to the proposed scheme as the SpaceTime Multilevel Sphere Packing Coded Modulation (STBCSP-MLC) arrangement.

The rest of this contribution is organized as follows. Section 2 provides an overview of the system considered, while our sphere packing aided iterative MSD assisted MLC based decoder is described in Section 3. Section 4 quantifies the performance of the STBC-SP-MLC scheme, characterizing its overall BER performance and its unequal error protection (UEP) capability, while our conclusions are presented in Section 5.

\section{SYSTEM OVERVIEW}

Figure 1 and 2 outline our proposed STBC-SP-MLC encoding and decoding scheme. A binary source bit stream $u$ is serial-to-parallel (S/P) converted, creating four individual bit protection levels, namely $u^{1}, u^{2}, u^{3}, u^{4}$, where the bits are protected by four different encoders. The output bits of encoder $C^{i}$, having a total encoded frame size of $n$ bits is denoted as $b^{i}=b_{1}^{i}, b_{2}^{i}, \ldots, b_{n}^{i}$. The bit interleaver $\pi$ of Figure 1 is optional, 


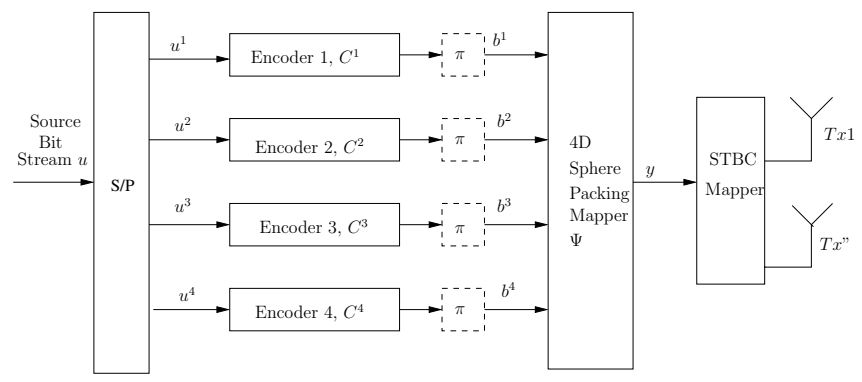

Figure 1: MLC encoder of the 4D, 4 bits/symbol (bps) sphere packing modulated symbol based STBC aided scheme.

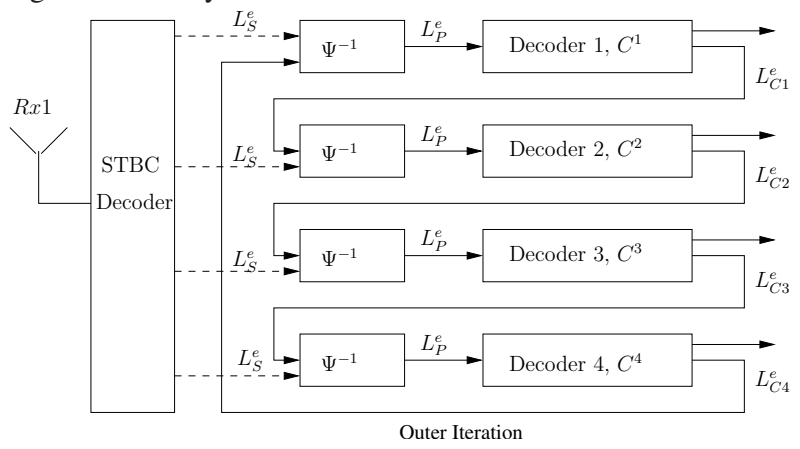

Figure 2: MSD Decoder of the 4D, 4bps sphere packing modulated symbol based STBC aided scheme.

depending on the specific construction of the individual component codes. In this paper, we employ Low-Density Parity Check (LDPC) component codes owing to their powerful iterative decoding and flexible coding rates. Each LDPC component code is decoded using the so-called belief propagation algorithm [10]. Due to the random nature of the parity check matrix construction of the LDPC code used, no additional interleaver is necessary in our system.

The coded bit streams $b^{1}, \ldots, b^{4}$ of Figure 1 are then converted into a 4-bit SP symbol, $y$, in the sphere packing modulator $\psi$. In our system, we consider a 4D SP scheme, which constitutes a natural design option, since we need a 4D SP modulation scheme having in excess of 16 SP constellation points. More specifically, the number of nearest points of a 4D sphere packing constellation is 24 [11] and we want to ensure that each of the 4-bit symbols $b^{1}, \ldots, b^{4}$ is mapped to a SP constellation point having the highest possible energy. Therefore, our choice of the specific $L=16$ points out of the entire set of 24 is based on finding the $L=16$ highest energy points.

The details of the SP-aided orthogonal design of a STBC scheme were provided in [9]. When using 4D SP symbols, again, in each dimension we have $L=16$ possible constellation points, namely the set of $4 \mathrm{D}$ phasor points of $S=\left(a_{l, 1}, a_{l, 2}, a_{l, 3}\right.$, $\left.a_{l, 4}\right)$, where $l=0,1,2, \ldots, L-1$. We consider the real-value of each SP phasor constellation point of the space $R^{4}$, where each of the four elements in $S$ represents a 4D coordinate point. The set $S$ would consist of all legitimate points having real coordinates satisfying the SP-constraint of $\left(a_{1}+a_{2}+a_{3}+a_{4}\right)=k$, where $k$ is an even integer. The total energy of these constella- tion points is given by $E \triangleq \sum_{l=0}^{L-1}\left(\left|a_{l, 1}\right|^{2}+\left|a_{l, 2}\right|^{2}+\left|a_{l, 3}\right|^{2}+\right.$ $\left|a_{l, 4}\right|^{2}$ ) [9]. Again, we conducted comprehensive investigations of different bit-to-SP-symbol mapping schemes and identified the ones, which maximized the minimum Euclidean distance of the entire $4 \mathrm{D}$ constellation space in our system. This particular mapping of the 4-bit tuples $b^{1}, \ldots, b^{4}$ to the $L=16$ 4D SP constellation points closely resembles Ungerböck's Set Partitioning (UP) concept [12]. The 4D SP symbol is split into two different complex-valued symbols, each hosting two coordinate values of the 4D SP symbol, before mapping them to Alamouti's STBC using two transmit antennas. Let $\rho($.$) be$ the SP mapping function from the original input bits and $\Gamma($.) represents the mapping of 4D SP symbol to complex-valued symbols before STBC. Hence, we have [9]

$$
\begin{aligned}
\Gamma\left(\rho\left(b_{1}, b_{2}, b_{3}, b_{4}\right)\right) & =\Gamma\left(a_{l, 1}, a_{l, 2}, a_{l, 3}, a_{l, 4}\right), \\
& =\left\{a_{l, 1}+j a_{a, 2}, a_{l, 3}+j a_{l, 4}\right\} .
\end{aligned}
$$

Each SP symbol is transmitted over the two antennas in two consecutive time slots, where the throughput of the combined SP-STBC arrangement is given by $\log _{2} L / 2$ bits per channel used. We fix our total system throughput to $1 \mathrm{bit} / \mathrm{channel}$ use in order to make a fair comparison between the STBC-SP-MLC scheme and other benchmarkers.

At the receiver side, a STBC decoder using a single receive antenna is employed, as shown in Figure 2. The set of complex-valued STBC symbols are forwarded to the SPdemodulator $\psi^{-1}$ and then to the soft iterative MSD, which invokes bit-by-bit decoding, for the sake of reducing the associated decoding complexity and for exchanging useful a prior $i$ information between both the SP demodulator and the individual LDPC decoders $C^{i}$ of the different MSD levels. The extrinsic Log-Likelihood Ratio (LLR) $L_{S}^{e}$ output by the STBC decoder is fed into the SP demodulator $\psi^{-1}$. As seen in Figure 2, initially the SP demodulator only receives the channel's output information from the STBC decoder, since no useful a priori information is available during the first decoding iteration at the output of the MSD. The extrinsic LLR $L_{P}^{e}$ output by the SP demodulator of Figure 2 consists of the entire sequence of LLR values representing the MLC codewords. Only the LLRs of the highest bit protection level are fed into the corresponding decoder $C^{1}$, where the LLR values were extracted from the set of $L_{P}^{e}$ seen in Figure 2. Observed in Figure 2 that the decoder's output forwards a set of corresponding extrinsic LLR values $L_{C 1}^{e}$ to the demodulator again. This useful information provides the a priori information for the SP demodulator. Only the LLRs from the first protection level are updated, while the rest remain zero in $L_{S}^{e}$. As the decoding process proceeds to the next MSD level, each MSD level receives new LLR information from the previous MSD levels. The next outer iteration exchanging information between the SP demodulator and the MLC decoders commences after the lowest protection level's decoder $C^{4}$ provided its LLR $L_{C 4}^{e}$ for the SP demodulator, where the entire LLR information of the SP demodulator is updated by taking into account all of its previous information from all different levels of the MSD scheme. 
The proposed system was investigated, when communicating over a narrowband Rayleigh fading channel, associated with a normalised Doppler frequency of $f_{D}=0.1$. The complex fading envelope spanning across two consecutive timeslot was assumed to be constant. The Additive White Gaussian Noise (AWGN) of $n=n_{I}+j n_{Q}$ was superimposed on the received signal, where $n_{I}$ and $n_{Q}$ represents the independent real and imaginary components of the zero-mean Gaussian noise. The AWGN has a variance of $\sigma^{2}=\sigma_{n_{I}}^{2}=\sigma_{n_{Q}}^{2}=N_{0} / 2$.

\section{ITERATIVE MULTISTAGE SPHERE PACKING DECODING}

The received SP symbol $r$ output by the STBC decoder can be written as [9]

$$
\mathbf{r}=h \cdot \sqrt{\frac{2 L}{E}} \cdot \mathbf{s}^{1}+\mathbf{w},
$$

where $h=\left(\left|h_{1}\right|^{2}+\left|h_{2}\right|^{2}\right), h_{1}$ and $h_{2}$ represent the channel impulse response (CIR) corresponding to the first and second transmit antennas, $\mathbf{s}^{\mathbf{l}} \in S, 0 \leq l \leq L-1$, and $\mathbf{w}$ is a $4 \mathrm{D}$ Gaussian random variable having a variance of $\sigma_{w}^{2}=h \cdot \sigma_{n}^{2}$. The conditional PDF of the Gaussian distribution is given by

$$
\begin{aligned}
p\left(r / s^{l}\right) & =\frac{1}{\left(2 \pi \sigma_{w}^{2}\right)^{\frac{N_{D}}{2}}} e^{-\frac{1}{2 \sigma_{w}^{2}}\left(r-\alpha \cdot \mathbf{s}^{1}\right)^{2}}, \\
& =\frac{1}{\left(2 \pi \sigma_{w}^{2}\right)^{\frac{N_{D}}{2}}} e^{-\frac{1}{2 \sigma_{w}^{2}}\left(\sum_{i=1}^{4}\left(\tilde{a}_{i}-\alpha \cdot a_{i}\right)^{2}\right)},
\end{aligned}
$$

where we have $N_{D}=4$ for the $4 \mathrm{D}$ sphere packing symbols associated with $\alpha=h \cdot \sqrt{\frac{2 L}{E}}$.

For the max-log approximation of the extrinsic LLR of a single bit $b_{k}$ output by the SP demodulator we have

$$
\begin{aligned}
& L\left(b_{k} / r\right)-L_{a}\left(b_{k}\right) \\
= & \ln \frac{\sum_{\mathbf{s}^{1} \in S_{1}^{k}} \exp \left[-\frac{1}{2 \sigma_{w}^{2}}\left(r-\alpha \cdot \mathbf{s}^{\mathbf{l}}\right)^{2}+\sum_{j=0, j \neq k}^{B-1} b_{j} L_{a}\left(b_{j}\right)\right]}{\sum_{\mathbf{s}^{1} \in S_{0}^{k}} \exp \left[-\frac{1}{2 \sigma_{w}^{2}}\left(r-\alpha \cdot \mathbf{s}^{\mathbf{l}}\right)^{2}+\sum_{j=0, j \neq k}^{B-1} b_{j} L_{a}\left(b_{j}\right)\right]} \\
= & \max _{\mathbf{s}^{\mathbf{1}} \in S_{1}^{k}}\left[-\frac{1}{2 \sigma_{w}^{2}}\left(r-\alpha \cdot \mathbf{s}^{\mathbf{l}}\right)^{2}+\sum_{j=0, j \neq k}^{B-1} b_{j} L_{a}\left(b_{j}\right)\right] \\
- & \max _{\mathbf{s}^{1} \in S_{0}^{k}}\left[-\frac{1}{2 \sigma_{w}^{2}}\left(r-\alpha \cdot \mathbf{s}^{\mathbf{l}}\right)^{2}+\sum_{j=0, j \neq k}^{B-1} b_{j} L_{a}\left(b_{j}\right)\right],
\end{aligned}
$$

where the SP symbols carry $B$ number of MLC bits, $\mathbf{b}=$ $b_{0, \ldots, B-1} \in\{0,1\}$. Let us assume furthermore that $S_{1}^{k}$ and $S_{0}^{k}$ are subsets of the SP symbol constellation $S$ such that $S_{1}^{k} \triangleq$ $\left\{\mathbf{s}^{\mathbf{l}} \in S: b_{k}=1\right\}$ and likewise, $S_{0}^{k} \triangleq\left\{\mathbf{s}^{\mathbf{l}} \in S: b_{k}=0\right\}$.

Let us also assume that we have $q$ different MLC protection levels and the MLC-encoded bits are mapped to a total of $N=2^{q}$ possible SP symbols. At the bit protection level $i$, the updated a priori information obtained from the preceding higher-protection MLC constituent decoder is

$$
L_{C i}^{e} \triangleq\left\{L_{a}\left(b_{k}\right) ; k \in\{t q+(i-1), t=0,1, \ldots, N\}\right\} .
$$

Our benchmarker system employs a MLC scheme using MSD and classic $\mathrm{M}=16$-ary modulation. The extrinsic probability of this MLC demapper used by the multi-stage decoding is [12]

$$
\begin{aligned}
P_{e}\left(b_{t}^{i}=b\right) & =\frac{\left(\sum_{y_{t} \in \chi(i, b)} P\left(b_{t} \mid y_{t}\right) P\left(y_{t}\right)\right)}{P\left(b_{t}^{i}=b\right)}, \\
& =\sum_{y_{t} \in \chi(i, b)}\left(P\left(b_{t} \mid y_{t}\right) \prod_{j \neq i} P_{a}\left(b_{t}^{j}=b^{j}\left(y_{t}\right)\right)\right),
\end{aligned}
$$

where $b \in\{0,1\}$ and $y_{t}$ is the output of the $\mathrm{M}=16$-ary modulator. For 16QAM, we have $y_{t} \in \chi$, where $\chi(i, b)=\left\{\mu\left(b^{0}, b^{1}, b^{2}\right.\right.$, $\left.\left.b^{3}\right) \mid b_{i}=b ; b_{j} \in 0,1, j \neq i\right\}$. The specific bit-to-symbol mapping function of the QAM benchmarker is defined by the function $\mu($.$) while the extrinsic LLR value is computed as$ $\ln \left\{P_{e}\left(b_{t}^{i}=1\right) / P_{e}\left(b_{t}^{i}=0\right)\right\}$. For the MSD scheme employing a conventional 16QAM demodulator, the conditional PDF of Equation 3 provides a value of $N_{D}=2$ and $\sigma_{w}$ represents the variance of the complex-valued 2D Gaussian random variable. These two systems will be compared in Section 4.

\section{SIMULATION RESULTS}

In this section we characterise the achievable BER performance of our proposed STBC-SP-MLC scheme outlined in Figures 1 and 2, when communicating over a Rayleigh fading channel having a Doppler frequency of 0.1 . We construct a few benchmarkers for comparison to the proposed scheme.

Firstly, the overall coding rate of our proposed STBC-SPMLC scheme is given by

$$
R_{s y s}=\frac{\sum_{i=1}^{i=q} k_{i}}{\sum_{i=1}^{i=q} n_{i}} \cdot \frac{N_{s y m} \cdot b p s_{s p}}{T_{r}},
$$

where $k_{i}$ and $n_{i}$ are the number of source bits and encoded bits of the individual MLC component codes, $T_{r}$ is the total number of STBC timeslots used for transmitting the associated pair of symbols, while $N_{\text {sym }}$ is the number of SP symbols at the input of the STBC encoder in a particular time slot and $b p s_{s p}$ is defined as the number of bits per SP symbol. Hence

(4) the total throughput of the system is $1 \mathrm{bit} / \mathrm{channel}$ use. Our system parameters are summarised in Table 1.

The coding rate based on classic 16QAM with largest Euclidean distance is chosen [5]. We use the same code rates as in [5], which has a similar construction nature of our SP model. The individual LDPC code rates are represented by those of Scheme 1 in Table 1. Each LDPC component code has a block length of 640 and their resultant total MLC coding rate is $0.49609(\approx 1 / 2)$. A total of 30002560 -encoded-bit 


\begin{tabular}{|l||c|c|c|r|}
\hline Coding rate & $R_{1}$ & \multicolumn{1}{|c|}{$R_{2}$} & \multicolumn{1}{c|}{$R_{3}$} & \multicolumn{1}{c|}{$R_{4}$} \\
\hline \hline Scheme 1 & $100 / 640$ & $450 / 640$ & $360 / 640$ & $360 / 640$ \\
Scheme 2 & $180 / 640$ & $100 / 640$ & $390 / 640$ & $570 / 640$ \\
Scheme 3 & $48 / 640$ & $228 / 640$ & $84 / 640$ & $280 / 640$ \\
\hline \hline Sphere packing modulation & Largest Min. Euclidean \\
Conventional modulation & 16QAM, Ungerböck P. \\
MLC component block length & & 640 \\
STBC-SP-LDPC block length & & 2560 \\
No. of LDPC iterations & & 5 \\
LDPC column weight & & 3 \\
LDPC decoding field & & GF(2) \\
Total frame length & & 3000 symbols \\
Overall system throughput & & 0.1 \\
Doppler frequency & & 2 \\
No. of transmitters & & 1 \\
No. of receiver & & \\
\hline
\end{tabular}

Table 1: System parameters.

frames were transmitted for BER computation. Our benchmarker is based on a STBC-MLC structure, which is constituted by the direct serial concatenation of STBC and MLC without SP modulation. The STBC employs two transmit antennas, a single receive antenna and the MLC maps the output symbols into a 2D 16QAM Ungerböck Partitioning (UP) based modulator. The LDPC coding rates for this STBC-MLC UP 16QAM benchmarker are represented by those of Scheme 3 in Table 1, which were obtained by applying the capacity rules derived for UP-aided 16QAM at a code rate of $1 / 4$ in [6]. The overall throughput of the system remains $1 \mathrm{bit} / \mathrm{channel}$ use. Additionally, we compare the beneficial effects of the STBC on the MLC schemes' performance by comparing the attainable BER performance to that of a pure MLC scheme operating without the aid of a serially concatenated STBC arrangement. The same coding rates are used, as detailed in the context of Scheme 3 of Table 1 .

Figure 3 illustrates the attainable BER performance of the proposed STBC-SP-MLC scheme to that of the two benchmarkers. Observe from the results of Figure 3 that the conventional MLC scheme does not perform well in a Rayleigh fading channel, exhibiting a BER just above $10^{-4}$ at $E_{b} / N_{0}=9 \mathrm{~dB}$ after $I=9$ iterations. The space diversity gain provided by a serially concatenated STBC scheme improves its BER performace in excess of an order of magnitude at the same $E_{b} / N_{0}$ value when using $I=5$ iterations. However, even this improved performance can be significantly enhanced with the aid of the proposed system employing the SP demapper. The BER curve dips below $10^{-4}$ using a single iteration at $E_{b} / N_{0}=5 \mathrm{~dB}$. Upon employing $I=9$ iterations, the BER is reduced to $2 \times 10^{-5}$ at the same $E_{b} / N_{0}$ value.

To elaborate a little further, a single-class $1 / 2$-rate STBCSP-LDPC scheme is also used for comparison with our MLC structure, where the MLC codes of Scheme 1 were replaced by the single-class $\operatorname{LDPC}(2560,1280)$ scheme of coding rate

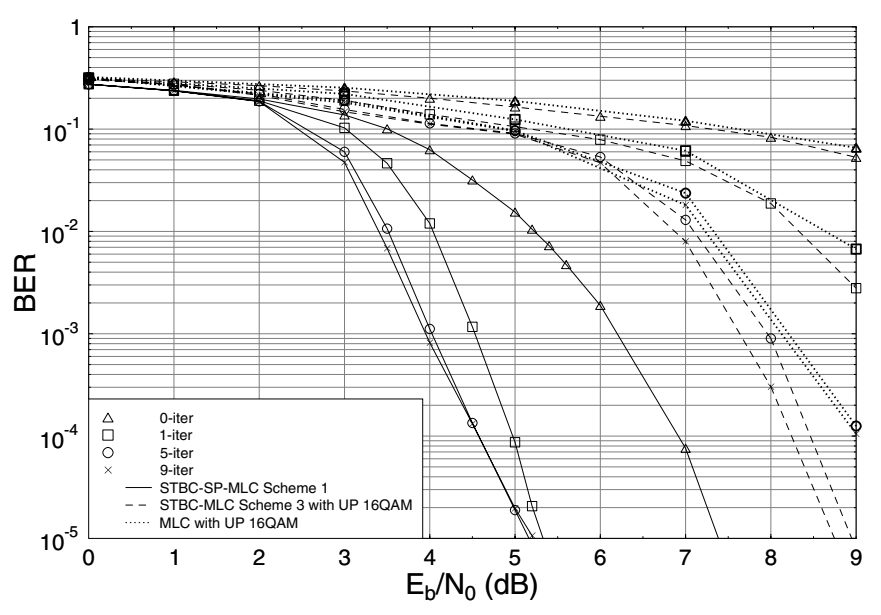

Figure 3: BER versus $E_{b} / N_{o}$ performance of the conventional MLC using 16QAM UP based mapping without STBC, the STBC-MLC 16QAM UP based mapping Scheme 3 of Table 1 and our proposed STBC-SP-MLC Scheme 1, communicating over a correlated Rayleigh channel.

1/2. All LDPC component codes employed in our simulations used a total of five iterations for generating reliable extrinsic LLRs. The complexity of a single 2560-coded-bit LDPC code and that of the four 640-coded-bit MLC-LDPC component codes was deemed similar in these systems. The LDPC decoding complexity of each iteration associated with a parity check matrix having a column weight of $j$ and row weight of $k$ may be approximated in terms of the number of additions and subtractions in the logarithmic domain [13] as comp $\{L D P C\}=(4 k+$ $j) j$. The corresponding BER results are shown in Figure 4.

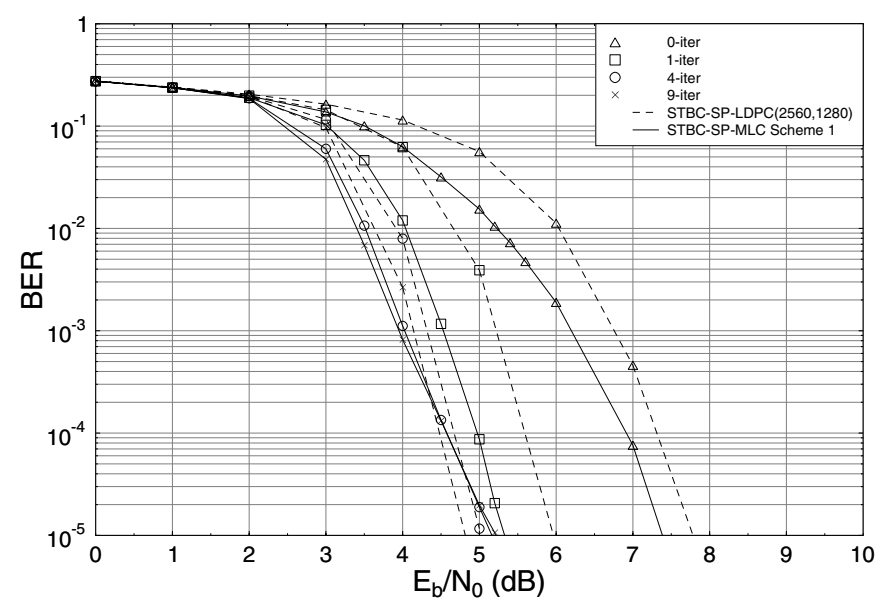

Figure 4: BER versus $E_{b} / N_{o}$ performance of STBC-SP-LDPC using a single 1/2-rate component code $\operatorname{LDPC}(2560,1280)$ in comparison to the proposed Scheme 1, communicating over a correlated Rayleigh fading channel.

Observe in Figure 4 that our proposed STBC-SP-MLC system exhibits a similar BER performance to that of the singleclass STBC-SP-LDPC structure, although the proposed scheme 
has a slightly better performance at a low number of iterations. By contrast, the single-class scheme performs approximately $0.5 \mathrm{~dB}$ better at a higher number of iterations at BER $10^{-5}$. This is a consequence of the fact that each MLC component code has a four times lower codeword length compared to the single $\operatorname{LDPC}(2560,1280)$ code. However, the advantage of using MLC is the shorter and hence lower-complexity component codes, the flexibility of freely adjusting the coding rates compared to other coded modulation schemes and its ability to protect each bit unequally as demonstrated in Figure 5.

\subsection{Unequal Error Protection}

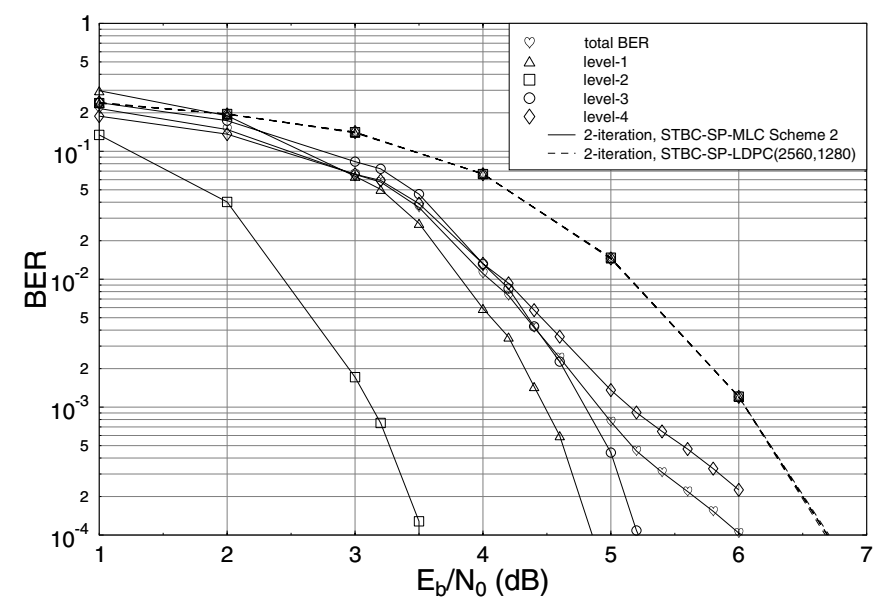

Figure 5: BER versus $E_{b} / N_{o}$ performance of STBC-SP-LDPC using a single 1/2-rate component $\operatorname{LDPC}(2560,1280)$ and the proposed STBC-SP-MLC Scheme 1, communicating over a correlated Rayleigh fading channel. Each bit protection level is shown as an individual BER curve.

The ability to provide unequal error protection is important for speech, audio and video applications, where each bit may have a different error sensitivity. For this type of applications, the single-class LDPC codes of the STBC-SP-LDPC scheme may be less favoured in comparison to the STBC-SPMLC scheme. Figure 5 illustrates the individual BER curves of both systems. All the bits in the single class STBC-SP-LDPC system exhibit a similar BER performance, as can be observed from the overlapping dashed curves in Figure 5.

A total of $I=2$ iterations were used by the MSD scheme. We adjusted the coding rate according to the partitioning regime of [5] using the so-called hybird $-I$ scheme in conjunction with 16QAM to strongly protect a small proportion of the most important bits using Scheme 2 of Table 1. Observe from the simulation results of Figure 5 that as expected, the individual BER performance of the different protection levels is quite different. Bit protection level-2 exhibits a high protection, associated with an $E_{b} / N_{0}$ improvement of up to $3 \mathrm{~dB}$ at $\mathrm{BER}=10^{-4}$, while maintaining a similar overall BER performance to that of the single-class STBC-SP-LDPC scheme.

\section{CONCLUSIONS}

In conclusion, a novel STBC-SP-MLC scheme was proposed, which invokes a multistage decoded MLC and SP arrangement combined with a concatenated STBC scheme. Our simulation results outlined in Figures 3, 4 and 5 illustrate that at $\mathrm{BER}=10^{-4}$ the proposed STBC-SP-MLC scheme is capable of achieving a significant $E_{b} / N_{0}$ improvement of about $3.5 \mathrm{~dB}$ and $4.5 \mathrm{~dB}$ compared to the STBC-MLC and the MLC benchmark system invoking 16QAM combined with a conventional MLC scheme. Even though this system may be slightly outperformed by a single-class STBC-SP-LDPC scheme at low BERs, the multiclass scheme exhibits a higher flexibility and unequal error protection capability. Our future research will investigate the capacity of STBC-SP-MLC and different code construction criteria for employment in the 4D SP space. Furthermore, rotationally invariant differential space-time coded schemes and various unequal-protection applications will be studied.

\section{REFERENCES}

[1] H. Imai and S. Hirawaki, "A New Multilevel Coding Method Using Error Crrecting Codes," IEEE Transactions on Information Theory, pp. 371377, May 1977.

[2] U. Wachsmann, R. F. H. Fischer and J. B. Huber, "Multilevel Codes: Theoretical Concepts and Practical Design Rules," IEEE Transaction on Information Theory, vol. 45, pp. 1361-1391, July 1999.

[3] S. M. Alamouti, "A Simple Transmitter Diversity Scheme for Wireless Communications," IEEE Journal on Selected Areas in Communications, vol. 16, pp. 1451-1458, October 1998.

[4] V. Tarokh, H. Jafarkhani and A.R. Calderbank, "Space-Time Block Codes from Orthogonal Designs," IEEE Transactions on Information Theory, vol. 45, pp. 1456-1467, July 1999.

[5] M. Isaka, R. H. M-Zaragoza, M. P. C. Fossorier, S. Lin and H. Imai, "Multilevel Coded 16-QAM Modulation with Multistage Decoding and Unequal Error Protection," Global Communication Conference, vol. 6, pp. 3548-3553, November 1998.

[6] L. H-J. Lampe, R. Schober and R. F. H. Fischer, "Multilevel Coding for Multiple-Antenna Transmission," IEEE Transactions on Wireless Communications, vol. 3, pp. 203-208, January 2004.

[7] D-F. Yuan, F. Zhang, A-F. So, Z-W. Li, "Concatenation of Space-Time Block Codes and Multilevel Coding over Rayleigh Fading Channels," (Atlantic City, USA), pp. 192-196, October Fall 2001.

[8] W. Su, Z. Safar, and K. J. R. Liu, "Space-Time Signal Design for Timecorrelated Rayleigh Fading Channels," (Anchorage, Alaska), pp. 31753179, May 2003.

[9] O. Alamri, B. L. Yeap, L. Hanzo, "Turbo Detection of Channel-Coded Space-Time Signals Using Sphere Packing Modulation," (Los Angeles, USA), pp. 2498-2502, September Fall 2004.

[10] R. Gallager, Low Density Parity Check Codes. USA: MIT Press, 1963.

[11] J. H. Conway and N. J. Sloane, "Sphere Packings, Lattices and Groups," Spinger-Verlag, 1999.

[12] L. Hanzo, T. H. Liew and B. L. Yeap, Turbo Coding, Turbo Equalisation and Space Time Coding for Transmission over Wireless channels. New York, USA: John Wiley IEEE Press, 2002.

[13] F. Guo, S. X. Ng and L. Hanzo, "LDPC assisted Block Coded Modulation for Transmission over Rayleigh Fading Channels," vol. 3, (Florida, USA), pp. 1867-1871, April Spring 2003. 